\title{
Bosonic molecules in rotating traps
}

\author{
Igor Romanovsky, Constantine Yannouleas, Leslie O. Baksmaty, and Uzi Landman \\ School of Physics, Georgia Institute of Technology, Atlanta, Georgia 30332-0430
}

(Dated: To appear in Phys. Rev. Lett.)

\begin{abstract}
We present a variational many-body wave function for repelling bosons in rotating traps, focusing on rotational frequencies that do not lead to restriction to the lowest Landau level. This wave function incorporates correlations beyond the Gross-Pitaevskii (GP) mean field approximation, and it describes rotating boson molecules (RBMs) made of localized bosons that form polygonal-ring-like crystalline patterns in their intrinsic frame of reference. The RBMs exhibit characteristic periodic dependencies of the ground-state angular momenta on the number of bosons in the polygonal rings. For small numbers of neutral bosons, the RBM ground-state energies are found to be always lower than those of the corresponding GP solutions, in particular in the regime of GP vortex formation.
\end{abstract}

PACS numbers: 05.30.Jp, 03.75.Hh

Recent experimental advances in the field of trapped ultracold neutral bosonic gases have enabled control of the strength of interatomic interactions over wide ranges [1, 2, 3, 4] , from the very weak to the very strong. This control is essential for experimental realizations of novel states of matter beyond the well known Bose-Einstein condensate 2, 3, 4]. In this context, the linear 1D TonksGirardeau regime of impenetrable trapped bosons has generated intensive theoretical activity [5, 6] and several experimental realizations of it have been reported most recently $[3,4]$.

Here we address the properties of strongly-repelling impenetrable bosons in rotating ring-shaped or $2 \mathrm{D}$ harmonic traps. To this end, we recall that impenetrable bosons are "localized" relative to each other [4, 7] and exhibit nontrivial intrinsic crystalline correlations [7]. For a small number of bosons, $N$, these crystalline arrangements are reminiscent of the structures exibited by the well-studied rotating electron molecules (REMs) in quantum dots under high magnetic fields [8, 9]. Consequently, we use in the following the term rotating boson molecules (RBMs). A central result of our study is that the point-group symmetries of the intrinsic crystalline structures give rise to characteristic regular patterns (see below) in the ground-state spectra and associated angular momenta of the RBMs as a function of the rotational frequency for neutral bosons (or the magnetic field for charged bosons).

An unexpected result of our studies is that the rotation of repelling bosons (even those interacting weakly) does not necessarily lead to formation of vortices, as is familiar from the case of rotating Bose-Einstein condenstates (BECs). In particular, for small $N$, we will show that the Gross-Pitaevskii energies (including those corresponding to formation of vortices) remain always higher compared to the ground-state energies of the RBMs. Of course, we expect that the rotating BEC will become the preferred ground state for sufficiently large $N$ in the case of weakly repelling neutral bosons. We anticipate, however, that it will be feasible to test our unexpected results for small $N$ by using rotating optical lattices, where it is established that a small finite number of atoms can be trapped per given site [2].

In a non-rotating trap, it is natural to describe a localized boson (at a position $\mathbf{R}_{j}$ ) by a simple displaced gaussian 7]. When the rotation of the trap is considered, the gaussian needs to be modified by a phase factor, determined through the analogy between the one-boson Hamiltonian in the rotating frame of reference and the planal motion of a charged particle under the influence of a perpendicular magnetic field $B$ (described in the symmetric gauge). That is, the single-particle wave function of a localized boson is

$\varphi_{j}(\mathbf{r}) \equiv \varphi\left(\mathbf{r}, \mathbf{R}_{j}\right)=\frac{1}{\sqrt{\pi} \lambda} \exp \left[\frac{\left(\mathbf{r}-\mathbf{R}_{j}\right)^{2}}{2 \lambda^{2}}-i \mathbf{r} \cdot\left(\mathbf{Q} \times \mathbf{R}_{j}\right)\right]$

with $\mathbf{Q} \equiv \hat{\mathbf{z}} /\left(2 \Lambda^{2}\right)$ and the width of the Gaussian $\lambda$ is a variational parameter; $\Lambda \equiv l_{B}=\sqrt{\hbar c /(e B)}$ for the case of a perpendicular magnetic field $\mathbf{B}$, and $\Lambda \equiv l_{\Omega}=$ $\sqrt{\hbar /(2 m \Omega)}$ in the case of a rotating trap with rotational frequency $\boldsymbol{\Omega}$. Note that we consider a $2 \mathrm{D}$ trap, so that $\mathbf{r} \equiv(x, y)$ and $\mathbf{R} \equiv(X, Y)$. The hamiltonian corresponding to the single-particle kinetic energy is given by $H_{K}(\mathbf{r})=(\mathbf{P}-\hbar \mathbf{Q} \times \mathbf{r})^{2} /(2 m)$, for the case of a magnetic field, and by $H_{K}(\mathbf{r})=(\mathbf{P}-\hbar \mathbf{Q} \times \mathbf{r})^{2} /(2 m)-m \Omega^{2} \mathbf{r}^{2} / 2$, for the case of a rotating frame of reference [10].

A toroidal trap with radius $r_{0}$ can be specified by the confining potential

$$
V(\mathbf{r})=\frac{\hbar \omega_{0}}{2}\left(r-r_{0}\right)^{n} / l_{0}^{n},
$$

with $l_{0}=\sqrt{\hbar /\left(m \omega_{0}\right)}$ being the characteristic length of the $2 \mathrm{D}$ trap. For $n \gg 2$ and $l_{0} / r_{0} \rightarrow 0$ this potential approaches the limit of a toroidal trap with zero width, which has been considered often in previous theoretical studies (see, e.g., Ref. 11]). In the following, we consider the case with $n=2$, which is more realistic from the experimental point of view. In this case, in the limit $r_{0}=0$, one recovers a harmonic trapping potential. 


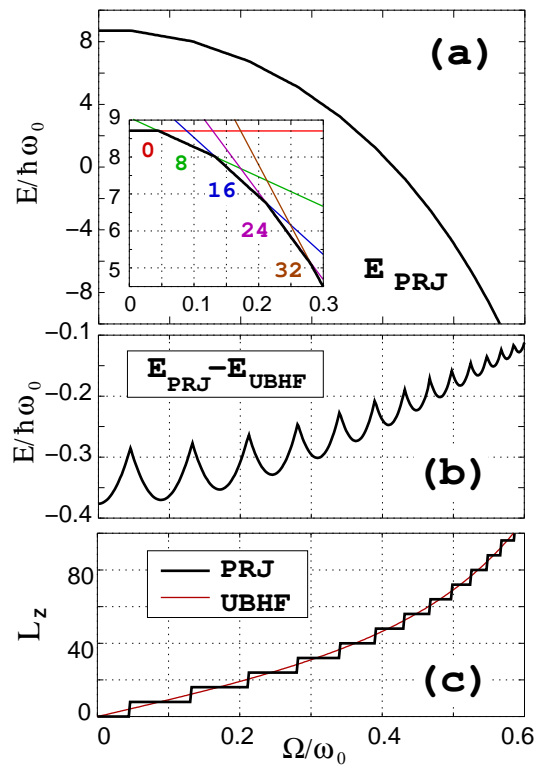

FIG. 1: Properties of $N=8$ neutral repelling bosons in a rotating toroidal trap as a function of the reduced rotational frequency $\Omega / \omega_{0}$. The confining potential is given by Eq. (2) with $n=2$ and radius $r_{0}=3 l_{0}$, and the interaction-strength parameter was chosen as $R_{\delta}=50$. (a) RBM ground-state energies, $E^{\mathrm{PRJ}}$. The inset shows the range $0 \leq \Omega / \omega_{0} \leq 0.3$. The numbers denote ground-state magic angular momenta. (b) Energy difference $E^{\mathrm{PRJ}}-E^{\mathrm{UBHF}}$. (c) Total angular momenta associated with (i) the RBM ground states [thick solid line (showing steps and marked as PRJ); online black] and (ii) the UBHF solutions (thin solid line; online red)

To construct an RBM variational many-body wave function describing $N$ impenetrable bosons in the toroidal trap, we use $N$ displaced orbitals $\varphi\left(\mathbf{r}, \mathbf{R}_{i}\right), i=$ $1,2, \ldots, N$ [see Eq. (1)] centered at the vertices of a regular polygon. Then, we first construct an unrestricted Bose Hartree-Fock (UBHF) permanent 7] $\left|\Phi_{N}^{\mathrm{UBHF}}\right\rangle \propto$ $\sum_{P\left(i_{m}\right)} \varphi_{1}\left(\mathbf{r}_{i_{1}}\right) \varphi_{2}\left(\mathbf{r}_{i_{2}}\right) \ldots \varphi_{N}\left(\mathbf{r}_{i_{N}}\right)$. The UBHF permanent breaks the circular symmetry of the many-body hamiltonian. The "symmetry dilemma" is resolved through a subsequent "symmetry-restoration" step accomplished via projection techniques [9, 12, 13], i.e., we construct a many-body wave function with good total angular momentum by applying the projection operator $\hat{\mathcal{P}}_{L}=$ $(1 / 2 \pi) \int_{0}^{2 \pi} d \theta \exp [i \theta(L-\hat{L})]$, so that the final RBM wave function is given by

$$
\left|\Psi_{N, L}^{\mathrm{PRJ}}\right\rangle=\frac{1}{2 \pi} \int_{0}^{2 \pi} d \theta\left|\Phi_{N}^{\mathrm{UBHF}}(\theta)\right\rangle e^{i \theta L} .
$$

$\left|\Phi_{N}^{\mathrm{UBHF}}(\theta)\right\rangle$ is the original UBHF permanent rotated by an azimuthal angle $\theta$. We note that, in addition to having good angular momenta, the projected (PRJ) wave function $\left|\Psi_{N, L}^{\mathrm{PRJ}}\right\rangle$ has also a lower energy than that of $\left|\Phi_{N}^{\mathrm{UBHF}}\right\rangle$ [see, e.g. $E_{L}^{\mathrm{PRJ}}-E^{\mathrm{UBHF}}$ in Fig. 1(b)]. The projected ground-state energy is given by

$$
E_{L}^{\mathrm{PRJ}}=\int_{0}^{2 \pi} h(\theta) e^{i \theta L} d \theta / \int_{0}^{2 \pi} n(\theta) e^{i \theta L} d \theta
$$

where $h(\theta)=\left\langle\Phi_{N}^{\mathrm{UBHF}}(\theta=0)|H| \Phi_{N}^{\mathrm{UBHF}}(\theta)\right\rangle$ and $n(\theta)=$ $\left\langle\Phi_{N}^{\mathrm{UBHF}}(\theta=0) \mid \Phi_{N}^{\mathrm{UBHF}}(\theta)\right\rangle$; the latter term ensures proper normalization.

The many-body hamiltonian is given by $\mathcal{H}=$ $\sum_{i=1}^{N}\left[H_{K}\left(\mathbf{r}_{i}\right)+V\left(\mathbf{r}_{i}\right)\right]+\sum_{i<j}^{N} V\left(\mathbf{r}_{i}, \mathbf{r}_{j}\right)$, with the interparticle interaction being given by a contact potential $V_{\delta}=g \delta\left(\mathbf{r}_{i}-\mathbf{r}_{j}\right)$ for neutral bosons and a Coulomb potential $V_{C}=Z^{2} e^{2} /\left|\mathbf{r}_{i}-\mathbf{r}_{j}\right|$ for charged bosons. The parameter that controls the strength of the interparticle repulsion relative to the zero-point kinetic energy is given by $R_{\delta}=g m /\left(2 \pi \hbar^{2}\right)$ [7 for a contact potential and $R_{W}=Z^{2} e^{2} /\left(\hbar \omega_{0} l_{0}\right)[7,14]$ for a Coulomb repulsion.

For a given value of the dimensionless rotational frequency, $\Omega / \omega_{0}$, the projection yields wave functions and energies for a whole rotational band comprising many angular momenta. In the following, we focus on the groundstate wave function (and corresponding angular momentum and energy) associated with the lowest energy in the band.

Fig. 1(a) displays the ground-state energy $E_{\mathrm{PRJ}}$ of $N=8$ bosons in a toroidal trap as a function of the dimensionless rotational frequency $\Omega / \omega_{0}$, with $\omega_{0}$ being the trap frequency. The prominent features in Fig. 1(a) are: (i) the energy diminishes as $\Omega / \omega_{0}$ increases; this is an effect of the centrifugal force, and (ii) the $E_{\mathrm{PRJ}}$ curve consists of linear segments, each one associated with a given angular momentum $L$. Most remarkable is the regular variation of the values of $L$ with a constant step of $N$ units (here $N=8$ ) [see inset in Fig. 1(a) and Fig. $1(\mathrm{c})]$. These preferred angular momenta $L=k N$ with integer $k$, are reminiscent of the so called "magic angular momenta" familiar from studies of electrons under high-magnetic fields in 2D semiconductor quantum dots [8, 9]. The preferred angular momenta reflect the intrinsic molecular structure of the localized impenetrable bosons. We note, that the $(0,8)$ polygonal-ring arrangement is obvious in the single-particle density associated with the UBHF permanent [see Fig. 2(b)]; $(0,8)$ denotes no particles in the inner ring and 8 particles in the outer one. After restoration of symmetry, however, the singleparticle (sp) density is circularly symmetric [see the PRJ sp-density in Fig. 2(c)] and the intrinsic crystallinity becomes "hidden"; it can, however, be revealed via the conditional probability distribution [7, 9] [CPD, see Fig. 2(d)]. We note the Gross-Pitaevskii sp-density in Fig. 2(a), which is clearly different from the PRJ density in Fig. 2(c).

The internal structure for charged bosons in a toroidal trap (not shown) is similar to that of neutral bosons (Fig. $2)$, i.e., a $(0,8)$ ring arrangement, portrayed also in the stepwise variation (in steps of 8 units) of the total angular 


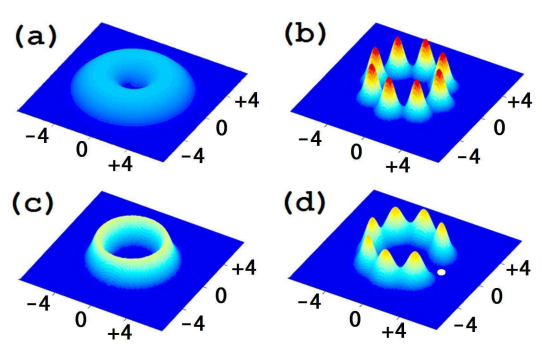

FIG. 2: Single-particle densities and CPDs for $N=8$ bosons in a rotating toroidal trap with $\Omega / \omega_{0}=0.2$ and $R_{\delta}=50$. The remaining trap parameters are as in Fig. 1. (a) GP sp-density. (b) UBHF sp-density exhibiting breaking of the circular symmetry. (c) RBM sp-density exhibiting circular symmetry. (d) $\mathrm{CPD}$ for the RBM wave function [PRJ wave function, see Eq. [3] revealing the hidden point-group symmetry in the intrinsic frame of reference. The observation point is denoted by a white dot. The RBM ground-state angular momentum is $L_{z}=16$. Lengths in units of $l_{0}$. The vertical scale is the same for (b), (c), and (d), but different for (a).

momenta. The internal structure is also reflected in the variation of the ground-state total energy as a function of the magnetic field. In contrast to the case of neutral bosons, however, the ground-state energy curve for charged bosons is not composed of linear segments, but of intersecting inverted-parabola-type pieces; this is due to the positive contribution of the Lorentz force compared to the negative contribution of the centrifugal force in a rotating trap.

For RBMs in rotating harmonic traps, the polygonalring pattern of localized bosons becomes more complex than the simple $(0, N)$ arrangement that appears naturally in a toroidal trap. Indeed, in harmonic traps, one anticipates the emergence of concentric ring structures. For $N=6$ neutral bosons in a harmonic trap, we observe that, as in the case of a toroidal trap, the groundstate energy as a function of the reduced rotational frequency, $\Omega / \omega_{0}$, [Fig. 3(a)] is composed of linear segments, but now the corresponding magic angular momenta [Fig. 3 (b)] vary in steps of $N-1=5$ units. This indicates an RBM consisting of two polygonal rings; denoted as a $(1,5)$ structure, with the inner ring having a single boson and the outer ring five.

In Fig. 4(a), we display the RBM and mean-field GP ground-state energies of $N=6$ strongly repelling (i.e., $R_{\delta}=50$ ) neutral bosons in a harmonic trap as a function of the reduced angular frequency of the trap. The GP curve (thin solid line; online red) remains well above the RBM curve (thick solid line; online green) in the whole range $0 \leq \Omega / \omega_{0} \leq 1$. The RBM ground-state angular momenta exhibit again the periodicity in steps of five units [Fig. 4(b)]. As expected, the GP total angular momenta are quantized $\left[L_{z}=0\right.$ (no-vortex) or $L_{z}=6$ (one central vortex)] only for an initial range $0 \leq \Omega / \omega_{0} \leq 0.42$. For $\Omega / \omega_{0} \geq 0.42$, the GP total angu- lar momentum takes non-integer values and ceases to be a good quantum number, reflecting the broken-symmetry character of the associated mean field, with each kink signaling the appearance of a different vortex pattern of $p$-fold symmetry $(p=1,2,3,4, \ldots)[15]$; see an example in Fig. 4(c).

The energetic superiority of the RBM wave function over the GP solution demonstrated in Fig. 4(a) was to be expected, since we considered the case of strongly repelling bosons. Unexpectedly, however, for a small number of neutral bosons the energetic advantage of the RBM persists even for weakly repelling bosons, as illustrated in Fig. 5(a). Indeed, Fig. 5(a) displays the RBM (thick solid line; online green) and GP (thin solid line; online red) ground-state energies for $N=6$ neutral bosons in a trap rotating with $\Omega / \omega_{0}=0.85$ as a function of the interaction parameter $R_{\delta}$. The surprising result in Fig. 5(a) is that the GP energy remains above the RBM curve even for $R_{\delta} \rightarrow 0$. Of course the RBM wave function is very close to that of a $\mathrm{BEC}$ without vortices when $R_{\delta} \rightarrow 0$ (BECs without vortices are approximately feasible for small $N$ ). However, for small $N$, our results show that BECs with vortices (i.e., for $L_{z} \geq N$ ) are not the preferred many-body ground states; instead, formation of RBMs is favored. Note that the energy difference $E^{\mathrm{GP}}-E^{\mathrm{PRJ}}$ increases rapidly with increasing $R_{\delta}$, reflecting the fact that the RBM energies saturate (as is to be expected from general arguments), while the GP ener-
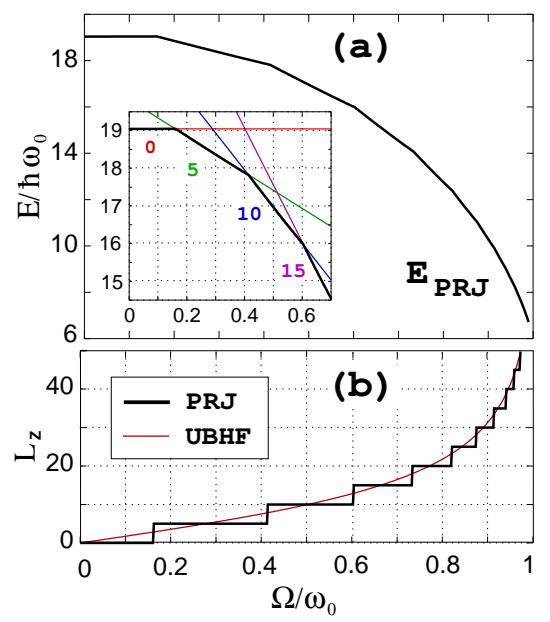

FIG. 3: Properties of $N=6$ neutral bosons in a rotating harmonic trap as a function of the reduced rotational frequency $\Omega / \omega_{0}$. The confining potential is given by Eq. (2) with $n=2$ and $r_{0}=0$, and the interaction-strength parameter was chosen as $R_{\delta}=50$. The intrinsic molecular structure is $(1,5)$. (a) RBM ground-state energies, $E^{\mathrm{PRJ}}$. The inset shows a smaller range. The numbers denote ground-state angular momenta. (b) Total angular momenta associated with (i) the RBM ground states (thick solid line showing steps; online black) and (ii) the UBHF solutions (thin solid line; online red). 

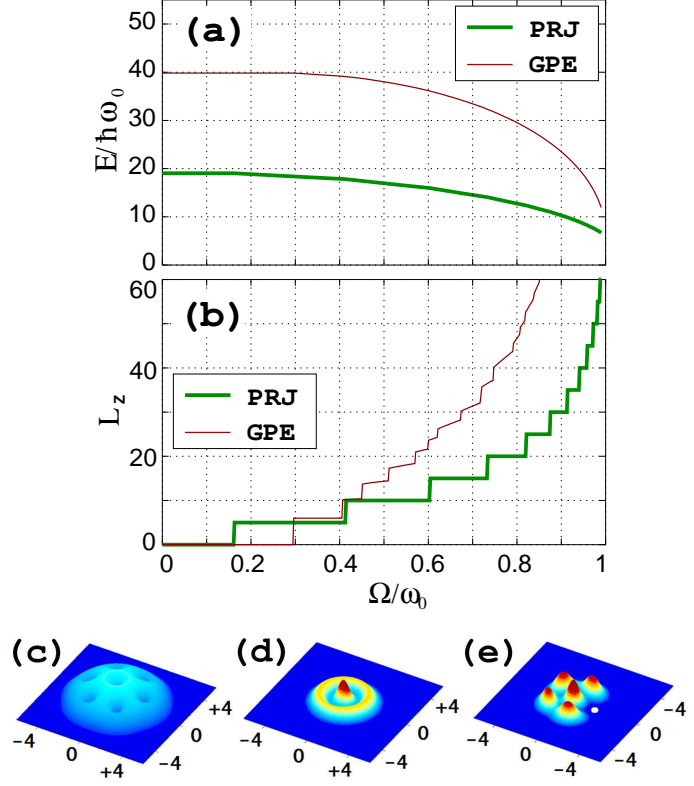

FIG. 4: Properties of GP solutions (thin solid line; online red) versus those of RBM wave functions (thick solid line; online green) for $N=6$ neutral bosons as a function of the reduced rotational frequency $\Omega / \omega_{0}$. A harmonic trap is considered, and the interaction strength equals $R_{\delta}=50$. (a) Ground-state energies. (b) Associated ground-state angular momenta. (c) GP (BEC) sp-density at $\Omega / \omega_{0}=0.65$ having 7 vortices with a 6 -fold symmetry (thus exhibiting breaking of the circular symmetry). (d) RBM sp-density at $\Omega / \omega_{0}=0.65$ which does not break the circular symmetry. (e) CPD of the $\mathrm{RBM}$ at $\Omega / \omega_{0}=0.65$ revealing the intrinsic $(1,5)$ crystalline pattern. The white dot denotes the observation point $\mathbf{r}_{0}$. Note the dramatic difference in spatial extent between the GP and RBM wave functions [compare (c) with (d) and (e). Lengths in units of $l_{0}$. The vertical scale is the same for (d) and (e), but different for (c).

gies (even with vortices fully accounted for) exhibit an unphysical divergence as $R_{\delta} \rightarrow \infty$ [Fig. 5(a)]; we have checked this trend up to values of $R_{\delta}=100$ (not shown). Of interest again is the different behavior of the RBM and GP ground state angular momenta [Fig. 5(b)] (see also discussion of Fig. 4(b)).

In conclusion, we have introduced (and studied the ground-state properties of) a variational many-body wave function for repelling bosons in rotating traps that incorporates correlations beyond the Gross-Pitaevskii mean-field approximations. This variational wave function describes rotating boson molecules, i.e., localized bosons arranged in polygonal-ring-type patterns in their intrinsic frame of reference. For small numbers of neutral bosons, and in particular in the case of GP vortex formation, the RBM ground-state energies are lower than those associated with the corresponding Gross-Pitaevskii BEC solutions. Given the large differences between the properties of the RBM and BEC wave functions (which become more pronounced for larger interaction parame-

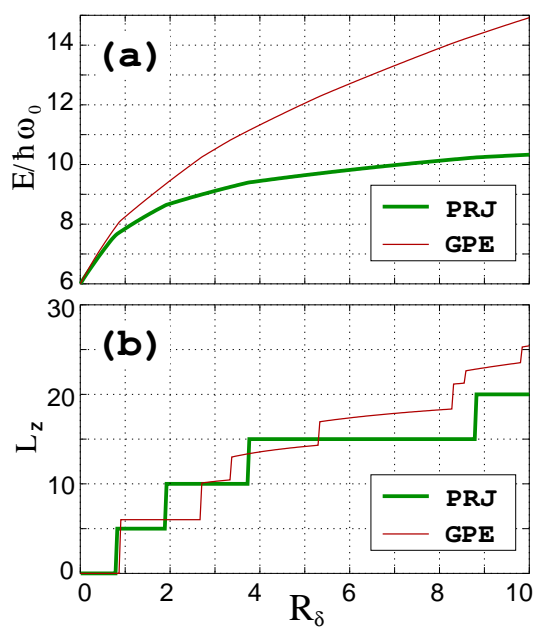

FIG. 5: Properties of GP solutions (thin solid line; online red) versus those of RBM wave functions (thick solid line; online green) for $N=6$ bosons as a function of the interaction strength $R_{\delta}$. A harmonic trap is considered, and the reduced rotational frequency equals $\Omega / \omega_{0}=0.85$. (a) Ground-state energies (b) Associated ground-state angular momenta.

ter $R_{\delta}$ ), and the recently demonstrated ability to experimentally control $R_{\delta}[1,2,3$, 4], we anticipate that our results could be tested in experiments involving rotating optical lattices. Detection of RBMs could be based on a variety of approaches [16], such as the measurement of the spatial extent [contrast the RBM and BEC spatial extents in Figs. 4(c)-4(e)], or the use of Hanbury Brown-Twiss-type experiments [17] to directly detect the intrinsic crystalline structure of the RBM.

This work is supported by the US D.O.E. (Grant No. FG05-86ER45234) and the NSF (Grant No. DMR0205328).

[1] S.L. Cornish et al., Phys. Rev. Lett. 85, 1795 (2000).

[2] M. Greiner et al., Nature (London) 415, 39 (2002).

[3] B. Paredes et al., Nature 429, 277 (2004).

[4] G.T. Kinoshita, T. Wenger, and D.S. Weiss, Science 305, 1125 (2004),

[5] M.D. Girardeau and E.M. Wright, Laser Physics 12, 8 (2002).

[6] V. Dunjko et al., Phys. Rev. Lett. 86, 5413 (2001).

[7] I. Romanovsky, C. Yannouleas, and U. Landman, Phys. Rev. Lett. 93, 230405 (2004).

[8] C. Yannouleas and U. Landman, Phys. Rev. B 68, 035326 (2003).

[9] C. Yannouleas and U. Landman, Phys. Rev. B 70, 235319 (2004); YS Li, C. Yannouleas, and U. Landman, Phys. Rev. B 73, 075301 (2006).

[10] The single-particle wave function in Eq. (1) and the many-body projected wave function in Eq. (3) contain contributions from higher Landau levels. These wave functions belong exclusively to the lowest Landau level 
(LLL) only in the limit when $\lambda=\sqrt{2} l_{B}$ in the case of a magnetic field, or $\lambda=\sqrt{2} l_{\Omega}$ and $\Omega / \omega_{0}=1$ in the case of a rotating trap. LLL investigations of neutral bosons using exact diagonalization (EXD) techniques have attempted to introduce analogies with the liquid-like bosonic Laughlin and composite-fermion wave functions [see e.g., Th. Jolicoeur and N. Regnault, Phys. Rev. B 70, 241307 (2004)]. Recently, however, the possibility of localization of neutral bosons in the LLL is also attracting attention. In particular, the EXD study in Ref. [16] shows that, for small $N$, formation of vortices in the LLL is not a prevalent phenomenon, and their appearance "is restricted to the vicinity of some critical values of the rotational frequency ..."

[11] P.F. Kartsev, Phys. Rev. A 68, 063613 (2003).
[12] P.-O. Löwdin, Rev. Mod. Phys. 34, 520 (1962); R.E. Peierls and J. Yoccoz, Proc. Phys. Soc. London, Sect. A 70, 381 (1957).

[13] C. Yannouleas and U. Landman, J. Phys.: Condens. Matter 14, L591 (2002); Phys. Rev. B 66, 115315 (2002).

[14] C. Yannouleas and U. Landman, Phys. Rev. Lett. 82, 5325 (1999).

[15] R.A. Butts and D.S. Rokhsar, Nature (London) 397, 327 (1999).

[16] A more detailed discussion on possible detection approaches of strongly correlated bosonic states is given in N. Barberan et al., Phys. Rev. A 73, 063623 (2006).

[17] M. Schellekens et al., Science 310, 648 (2005). 